\title{
More Light on Random Number Generators
}

coexist, with many cases of interference, intermittency, stability, and instability.

A conceptual framework which could transfer the experience gained in nuclear physics to other fields is still missing. Interdisciplinary experts are not so numerous, and there remains much to be done until solutions to many-body problems in nuclear physics could be useful in biology, sociology or economics. Nonetheless, it can be safely claimed that atomic nuclei, and more specifically nuclear collisions, provide a frame of reference for the study of complexity that is unparalleled in the richness of its phenomenology. It would be a serious mistake to overlook this opportunity.

[1] Haxel O., Jensen J.H.D. and Suess H.E., Phys. Rev. 75 (1949) 1766; Mayer M.G., Phys. Rev. 74 (1948) 235; ibid. 75 (1949) 1969.

[2] Bohr N. and Kalckar K., Mat. Fys. Medd. Dan. Vid. Selsk. 14 (1937) No. 10; Bohr A. Mat. Fys. Medd. Dan Vid. Selsk. 26 (1952) No. 14; A. Bohr A. and Mottelson B.R., Mat. Fys. Medd. Dan. Vid. Selsk. 27 (1953) No. 16.

[3] Twin P.J. et al., Phys. Rev. Lett. 57 (1986) 811.

[4] Hansen P.G. and Johnson B., Europhys. Lett. 4 (1987) 409.

[5] Kramers H.A., Physica VII 4 (1940) 284.

[6] Balian R. and Bloch C., Ann. Phys. (New York) 69 (1972) 76.

[7] Wigner E.P., Ann. Math. 62 (1955) 548.

[8] Bohigas O. and Weidenmüller H.A., Ann.

Rev. Nucl. Part. Sci. 38 (1988) 421.

[9] Jakobsson B. et al, Z. Physik A 307 (1982) 293.

[10] Bertsch G. and Das Gupta S., Phys. Rep. 160 (1988) 189.

[11] Ayik S. and Grégoire Ch., Phys. Lett. B 212 (1988) 269.

[12] Heiselberg H., Pethick C.J. and Ravenhall D.G., Phys. Rev. Lett. 61 (1988) 818.

[13] Botet R. and Ploszajczak M., Phys. Rev. Lett. 69 (1992) 3696.

[14] Campi X., J. Phys. A 19 (1986) L917; Phys. Lett. B 208 (1988) 351.

[15] Ploszajczak M. and Tucholski A., Phys. Rev. Lett. 65 (1990) 1539.
Considerable activity has been triggered by recent results of Monte Carlo calculations showing that random number generators considered good were giving the wrong answers in Ising model simulations using the highly efficient Wolff algorithm [see EN 24 (1993) 24].

Until recently, the only pseudorandom number generators which had been studied extensively were those of the multiplicative congruential type; they present a well-known defect whose effect can be made arbitrarily small at the expense of some computing time, and there is no wider theory which excludes their having additional as yet unknown defects. For this reason, many Monte Carlo practitioners prefer to use random numbers generated using other algorithms which produce much longer sequences before repeating and do not have the known defect of congruential generators. Unfortunately, Ferrenberg et al. [Phys. Rev. Lett. 69 (1992) 3382] showed that for certain problems these newer generators gave wrong answers. Now Martin Lüscher of DESY has studied one of these generators, the "subtract-with-borrow" algorithm of Marsaglia and Zaman known as RCARRY, and was able to show how to improve its chaotic properties.

As Lüscher says: "The characteristic feature of a chaotic dynamical system is that trajectories starting at nearby states diverge exponentially with time. Even if the evolution is locally continuous, such a system appears to behave randomly on larger time scales. One could also say that any state specified to some finite precision has an exponentially deteriorating memory of its history." Although RCARRY is "strongly chaotic" in this sense, it has short-term correlations which can be eliminated by a simple modification.

The RCARRY algorithm works on a table of 24 floating-point numbers and produces its next number by combining two of the numbers in the table and replacing one of them by the new result. Lüscher therefore

\section{Postdoctoral Fellowships \\ at the}

\section{Niels Bohr Institute}

The Niels Bohr Institute is part of the physics department of Copenhagen University and has active research programmes in theoretical and experimental particle physics, nuclear physics, physics of nonlinear and complex systems, cluster physics and theoretical and observational astrophysics. The Institute is host for two independent research institutes: Nordita (Nordic Institute for Theoretical Physics) and NBA (Niels Bohr Archive) and three research centres: TAC (Center for Theoretical Astrophysics), CATS (Center for Chaos and Turbulence Studies) and Connect (Center for Neural Network Studies), and there is a close collaboration with the scientists of these institutions. The Niels Bohr Institute has active programmes on research facilities such as the CERN accelerators, ESO and NOT telescopes, and the NORDBALL detector.

All applications for positions at the Niels Bohr Institute will be considered under the Institute's own international programmes. Applicants who are citizens of EC member states (or countries treated as member states) are eligible for fellowships at the "Niels Bohr Institute EC Physics Training Center". This is supported by the European Community "Human Capital and Mobility" programme.

Applicants should include a curriculum vitae, list of publications and a statement of their research interests and goals. In addition, applicants should arrange for 2-3 letters of reference to be sent directly. All material should be sent to:

Postdoctoral Positions Office, Ulla Holm, Secretary

The Niels Bohr Institute, Blegdamsvej 17, DK-2100 Copenhagen Ø, Denmark.

Tel: (+45) 35325272 - Fax: (+45) 31421016 - Email: uhclm@nbi.dk

The deadline for applications for the academic year 1994/95 is 12 December 1993 .

\section{RANLUX}

A subroutine implementing the skipping algorithm called RANLUX (for luxury random numbers) has been written by $F$. James and is being introduced into the CERN Program Library. The program, along with the paper of M. Lüscher [DESY preprint 93-133] will be published in Computer Physics Communications. Subroutine RANLUX allows the user to choose the "luxury level" between zero (no skipping, same as RCARRY) and four (skipping 365). Level two already passes all known tests and gives the right answers for the Wolff algorithm, but users who want an even better guarantee of security and can afford the extra computer time (about a factor 2.5 at level two and 5 at level four) can opt for pure luxury. For many problems, where the random number generation time is small compared with the rest of the calculation, there is no longer any reason for the computational physicist to deny himself the luxury of random numbers with demonstrably good chaotic properties.

looked at it as a generator of vectors in the 24-dimensional hypercube, and considered how two neighboring vectors in this hypercube would evolve according to the algorithm. He showed that indeed nearby trajectories would diverge exponentially, on the average with a Lyapunov exponent close to one, that is the separation would increase by about a factor $e$ every cycle of 24 numbers. After about 16 cycles, the smallest possible separation $\left(6 \times 10^{-8}\right)$ would grow to be of order one and fill all the hypercube.

In order to accelerate this "chaotic explosion", he suggested a modification of the algorithm whereby one would generate 24 numbers and then skip a certain number (at most 365 ) before using 24 more. This "throwing away" of random numbers is normally to be avoided since it shortens the effective period of a generator, but Lüscher also showed that if the total number generated and skipped per cycle is prime, the period is maintained. (In fact, since the basic period of RCARRY is about $10^{170}$, one can afford to shorten the period by many orders of magnitude without any danger of exhausting it.)

F. James, CERN, Geneva

\section{ERRATA}

Some mistakes crept into last month's anniversary issue of Europhysics News. The Editor apologises for the following:

- The group shown on the cover is the Conference Committee and not the Publications Committee.

- E. Gotsman from Tel Aviv University shown in the photo on p. 120 is clearly not a "Turkish politician".

- The Condensed Matter general conference had a "new beginning" in 1986 and not 1980 (p. 125).

- J.M. Charap was the Chairman and not the Secretary of High Energy Physics in 1981-84 (p. 126).

- Seated to the right of W. Buckel in the photo on p. 150 is L.A. Radicati and not G. Szigeti. 